\title{
Comparison of Unyvero P55 Pneumonia Cartridge, in-house PCR and culture for the identification of respiratory pathogens and antibiotic resistance in bronchoalveolar lavage fluids in the critical care setting
}

\author{
Naomi J. Gadsby ${ }^{1}$ (D) Martin P. McHugh ${ }^{1} \cdot$ Callum Forbes $^{2,3} \cdot$ Laura MacKenzie $^{1}$ - Stephen K. D. Hamilton ${ }^{2,4}$. \\ David M. Griffith ${ }^{2,5} \cdot$ Kate E. Templeton ${ }^{1}$
}

Received: 21 December 2018 / Accepted: 28 February 2019 / Published online: 11 March 2019

(C) Springer-Verlag GmbH Germany, part of Springer Nature 2019

\begin{abstract}
Faster respiratory pathogen detection and antibiotic resistance identification are important in critical care due to the severity of illness, significant prior antibiotic exposure and infection control implications. Our objective was to compare the performance of the commercial Unyvero P55 Pneumonia Cartridge (Curetis AG) with routine bacterial culture methods and in-house bacterial multiplex real-time PCR assays. Seventy-four bronchoalveolar lavage specimens from patients admitted to a Scottish intensive care unit (ICU) over a 33-month period were tested prospectively by routine culture and viral PCR and retrospectively by Unyvero P55 and in-house bacterial PCR. Sensitivity/specificity was 56.9\%/58.5\% and 63.2\%/54.8\% for the Unyvero P55 and in-house bacterial PCR panels respectively; sensitivity for in-panel targets was 63.5 and $83.7 \%$ respectively. Additional organisms were detected by Unyvero P55 and in-house bacterial PCR panels in 16.2\% specimens. Antibiotics were changed on the basis of routine test results in $48.3 \%$ cases; of these, true-positive or true-negative results would have been obtained earlier by Unyvero P55 or in-house bacterial PCR panel in $15(53.6 \%)$ and 17 (60.7\%) cases respectively. However, a false-negative molecular test result may have been acted upon in six (21.4\%) cases with either assay. Sensitivity/specificity of Unyvero P55 antibiotic resistance detection was $18.8 \% / 94.9 \%$ respectively. Molecular testing identified a number of respiratory pathogens in this patient cohort that were not grown in culture, but resistance detection was not a reliable tool for faster antibiotic modification. In their current set-up, molecular tests may only have benefit as additional tests in the ICU pneumonia setting.
\end{abstract}

Keywords Pneumonia $\cdot$ Bacteria $\cdot$ PCR $\cdot$ Antibiotic resistance $\cdot$ Critical care

Electronic supplementary material The online version of this article (https://doi.org/10.1007/s10096-019-03526-x) contains supplementary material, which is available to authorized users.

Naomi J. Gadsby

naomi.gadsby@nhslothian.scot.nhs.uk

1 Medical Microbiology, Department of Laboratory Medicine, Royal Infirmary of Edinburgh, Edinburgh EH16 4SA, UK

2 Critical Care, Royal Infirmary of Edinburgh, Edinburgh EH16 4SA, UK

3 Present address: Anaesthetics Department, St Johns Hospital, Livingston EH54 6PP, UK

4 Present address: Anaesthetics Department, James Cook University Hospital, Middlesbrough TS4 3BW, UK

5 Departmant of Anaesthesia, Critical Care and Pain Medicine, University of Edinburgh, Edinburgh EH16 4SA, UK

\section{Introduction}

Faster respiratory pathogen detection and antibiotic resistance identification are important in critical care due to the severity of illness, significant prior antibiotic exposure and infection control implications. Many respiratory viral and atypical bacterial panel assays are commercially available and offer rapid molecular detection for both high- and low-throughput laboratory and near-patient test settings [1,2]. However, rapid, automated commercial molecular assays for the typical respiratory bacteria targeted by most broad-spectrum empirical antibiotic regimens are significantly fewer in number and lack important antibiotic resistance gene targets [3, 4]. Therefore, antibiotic de-escalation and pathogen-targeted antimicrobial therapy for severe pneumonia identified in the intensive care unit (ICU) still relies on the results of slow, traditional microbiological culture methods. 
We recently developed in-house fast real-time quantitative PCR assays for typical respiratory bacteria [5] and demonstrated that a comprehensive molecular testing approach for sputum in community-acquired pneumonia (CAP) significantly improved microbiological diagnosis and had the potential to enable early pathogen-directed antibiotic therapy [6]. However, this approach requires a number of individual multiplex reactions for each specimen and does not incorporate antibiotic resistance gene detection. The Unyvero P55 Pneumonia Cartridge (Curetis AG) is a commercially available rapid molecular assay which can detect 21 respiratory pathogen species and genera alongside 17 antibiotic resistance genes [7]. The test uses an automated sample-to-answer format with minimal hands-on time and takes approximately $5 \mathrm{~h}$, with the potential to be used as a point-of-care test. Targets comprise two Gram-positive bacteria, 15 Gram-negative bacteria and four agents of atypical pneumonia: Legionella pneumophila, Mycoplasma pneumoniae, Chlamydophila pneumoniae and Pneumocystis jirovecii.

The aim of the study was to compare the performance of the Unyvero P55 Pneumonia Cartridge to standard culturebased methods and our in-house bacterial molecular diagnostic assays on bronchoalveolar lavage fluids (BALs) from ICU patients. We also intended to estimate the potential impact of rapid molecular assays on antibiotic prescribing in this setting.

\section{Methods}

\section{Specimens and patients}

Seventy-four BAL fluids from 74 consecutive individual patients admitted to the Royal Infirmary of Edinburgh ICU between 1 January 2013 and 30 September 2015 were tested using routine microbiological culture and molecular methods. Residual specimens were diluted approximately $1 / 10$ in UTM viral transport medium (Copan) and stored at $-80{ }^{\circ} \mathrm{C}$. Microbiology test results were obtained from the laboratory database for each BAL sample and combined with anonymised clinical data and information on antimicrobial prescription. Suspected ventilator-associated pneumonia (VAP) was defined as the presence of clinical and radiological signs of pneumonia in a patient mechanically ventilated for $\geq$ $48 \mathrm{~h}$. Suspected hospital-acquired pneumonia (HAP) was defined as the presence of clinical and radiological signs of pneumonia in a patient hospitalised for $\geq 48 \mathrm{~h}$. Suspected community-acquired pneumonia (CAP) was defined as the presence of clinical and radiological signs of pneumonia in a patient hospitalised for $<48 \mathrm{~h}$. Study approval was granted by NHS Lothian Caldicott Guardian and by NHS Lothian SAHSC Bioresource (SR211), with ethical approval to test anonymised, excess patient samples (10/S1402/33).

\section{Prospective testing}

Standard microbiological culture, biochemical and/or MALDI-TOF (Bruker) identification and automated antibiotic sensitivity testing (VITEK2, bioMérieux) were carried out according to national standard protocols [8]. All specimens were also tested by viral and atypical agent real-time PCRs adapted from previously published assays or developed inhouse [9-14]. CMV real-time PCR was performed routinely on all specimens using the Sentosa SA CMV Quantitative PCR Test (Vela Diagnostics). Total nucleic acid was extracted from specimens using the automated nucliSENS easyMAG (bioMerieux) system with off-board lysis, input volume of $200 \mu \mathrm{l}$ and elution volume of $100 \mu \mathrm{l}$. The same extract was used in multiplex real-time PCR assays in a panel comprising: (1) influenza A, influenza B and respiratory syncytial virus; (2) parainfluenza viruses 1, 2 and 3; (3) adenovirus and Mycoplasma pneumoniae; (4) rhinovirus and human metapneumovirus; (5) Legionella species and L. pneumophila; (6) CMV and (7) HSV-1 and 2. Real-time PCR for Pneumocystis jirovecii was carried out following specific clinical request.

\section{Retrospective testing}

Thawed BAL specimens were tested using both commercial and in-house bacterial respiratory panels after a period of storage at $-80^{\circ} \mathrm{C}$ ranging from 8 to 41 months. For the Unyvero P55 pneumonia assay (Curetis AG), specimens were processed according to the manufacturer's instructions and two specimens were processed simultaneously. A $180 \mu$ l specimen was added to the sample tube, placed into the lysator for $30 \mathrm{~min}$ and then placed into the test cartridge and loaded into the analyser. Targets for the Unyvero P55 assay are as follows: Staphylococcus aureus, Streptococcus pneumoniae, Citrobacter freundii, Escherichia coli, Enterobacter cloacae complex, Enterobacter aerogenes, Proteus spp., Klebsiella pneumoniae, K. oxytoca, K. variicola, Serratia marcescens, Morganella morganii, Moraxella catarrhalis, Pseudomonas aeruginosa, Acinetobacter baumannii complex, Stenotrophomonas maltophilia, Haemophilus influenzae, L. pneumophila, M. pneumoniae, C. pneumoniae, P. jirovecii and resistance genes: ermB, mecA, mecC, tem, shv, ctx-M, kpc, imp, ndm, oxa-23, oxa-24/40, oxa-48, oxa-58, vim, sul1, gyrA83 and gyrA87. For in-house respiratory bacteria multiplex real-time PCR, $200 \mu \mathrm{l}$ specimen was treated with lysozyme at $37^{\circ} \mathrm{C}$ followed by proteinase $\mathrm{K}$ at $56^{\circ} \mathrm{C}$ for $1 \mathrm{~h}$ each. Total nucleic acid was then extracted using the automated nucliSENS easyMAG (BioMérieux) instrument with an offboard lysis protocol, including the addition of phocine herpesvirus as an internal extraction control. Fast quantitative realtime PCR was performed using two multiplex assays developed for research use: (1) S. pneumoniae, H. influenzae, 
M. catarrhalis, S. aureus and (2) E. coli, K. pneumoniae, P. aeruginosa, A. baumannii [5].

\section{Statistical analysis}

Two-by-two contingency tables of categorical variables were analysed by either Fisher's exact test or chi-square with Yates' correction (GraphPad, CA, USA) to assess the effect of antibiotic exposure on test positivity. For sensitivity and specificity calculations, routine culture methodology was considered to be the gold standard.

\section{Results}

\section{Patient characteristics}

BAL fluids were available for retrospective testing from 74 patients admitted to the intensive care unit during the study period. Patients were admitted to the unit for a wide variety of reasons, reflecting the hospital's function as a major tertiary care centre (Table 1). However, BAL was performed predominantly as part of the diagnostic workup for pneumonia, particularly ventilator-associated pneumonia (Table 1).

\section{Prospective BAL fluid testing}

BAL fluids available for the study were equally distributed over the 33 month study period with $29 / 74(39.2 \%)$ in 2013 , $24 / 74(32.4 \%)$ in 2014 and $21 / 74(28.4 \%)$ in 2015. Prospective microbiological testing by standard culture and viral and atypical PCR screening identified an organism in $70 / 74(94.6 \%)$ specimens, with more than one organism reported in 46/74 (62.2\%) specimens (Table 2). Bacteria were isolated by routine culture in $48 / 74(65 \%)$ specimens, with Gram-negative bacteria in $42 / 74(56.8 \%)$ specimens. Of the 17 bacterial species cultured, 11/17 (64.7\%) were present in the Unyvero P55 panel and 6/17 (35.3\%) were present in the in-house PCR panel. Yeasts were the most frequently isolated group of fungal organisms, being present in 30/74 (40.5\%) specimens, whilst viruses were detected in $21 / 74(28.4 \%)$ specimens.

\section{Retrospective molecular testing}

Compared to the bacterial culture gold standard, overall sensitivity/specificity was $56.9 \% / 58.5 \%$ for the Unyvero P55 panel and $63.2 \% / 54.8 \%$ for the in-house PCR panel (Table 3). Cultured bacteria were not detected by the Unyvero P55 and in-house PCR panel in 25 (33.8\%) and 21 (28.4\%) specimens respectively (Table 3). For Unyvero P55, 19/25 (76.0\%) missed detections were clinically significant bacteria present in the panel. For in-house PCR, 7/21
(33.3\%) missed detections were clinically significant bacteria present in the panel and 9/21(42.9\%) were clinically significant bacteria not present in the panel (Table S1). Single isolates of six rare respiratory or clinically non-significant organisms were not detected by either molecular assay as targets were not present in either panel (Table S1). Additional organisms were detected by Unyvero P55 and in-house PCR panels in $12(16.2 \%)$ and $12(16.2 \%)$ specimens respectively (Table 3 and Table S2). The Unyvero P55 panel made 17 additional bacterial detections; $8 / 10(80 \%)$ of these with corresponding panel targets were also positive by in-house PCR. The inhouse PCR panel made 19 additional bacterial detections; 8/ $19(42.1 \%)$ of these were also positive by Unyvero P55 (Table S2).

Compared to the bacterial culture gold standard, in-panel sensitivity/specificity was $63.5 \% / 58.5 \%$ for the Unyvero P55 panel and $83.7 \% / 54.8 \%$ for the in-house PCR panel (Table 3 ).

\section{Antibiotic susceptibility}

Unyvero P55 antibiotic susceptibility prediction by the presence or absence of resistance genes was compared to the gold standard automated culture-based phenotypic testing, giving a sensitivity of $18.8 \%$, specificity of $94.9 \%$, NPV of $74.2 \%$ and a non-calculable PPV (Table 4). In particular, phenotypic amoxicillin, third-generation cephalosporin and macrolide/ lincosamide resistance were poorly detected by the molecular test, with $8 / 25(32 \%), 0 / 8(0 \%)$ and $0 / 8(0 \%)$ resistant isolates detected respectively. Furthermore, of the five instances of fluoroquinolone resistance detection by molecular assay, four $(80 \%)$ were false positive. There were an additional 12 instances of resistance gene detection where the corresponding antibiotic susceptibility phenotype was unknown. This was due to either the antibiotic not being present in the phenotypic battery tested or no growth from the specimen: these were sull (6), mecA (3), ermB (2) and tem (1).

\section{Antibiotic prescribing}

Data on antibiotic prescription in ITU were available for 58 (78.4\%) patients in the study. On the day of the bronchoalveolar lavage procedure, $37 / 58(63.8 \%)$ patients were receiving at least one antibiotic. The frequency of bacterial detection by routine culture was significantly higher in patients not receiving antibiotics $(18 / 22,81.8 \%)$ than in those receiving antibiotics $(18 / 36,50.0 \%)$, (difference $31.8 \%$ (95\% CI 6.2 to $50.8 \%$ $(p=0.025))$. Detection frequency was not affected by antibiotic prescription for either the Unyvero P55 panel (12/35 $(34.3 \%)$ vs. $11 / 22(50.0 \%)$ respectively, $p=0.368)$ or the inhouse PCR panel (17/36 (47.2\%) vs.10/22 (45.5\%) respectively, $p=0.896)$. The turnaround time from electronic test request to final electronic reporting of all prospective microbiological investigations on the BAL fluid sample was a 
Table 1 Characteristics of included patients $(n=74)$ with BAL fluid tested in the study

\begin{tabular}{|c|c|}
\hline Demographics & No. $(\%)$ \\
\hline Male $(\%)(n=74)$ & $44(59.5)$ \\
\hline Age, mean [stdev] years $(n=74)$ & $56.6[16.3]$ \\
\hline \multicolumn{2}{|l|}{ ITU admission diagnosis $(n=74)$} \\
\hline Recovery post- GI/hepatobiliary/cardiothoracic surgery & $14(18.9)$ \\
\hline Respiratory failure of non-infective/unknown cause & $10(13.5)$ \\
\hline Cardiac arrest & $7(9.5)$ \\
\hline Severe CAP/HAP & $7(9.5)$ \\
\hline Sepsis/septic shock & $7(9.5)$ \\
\hline Chest source & 2 \\
\hline Abdominal source & 1 \\
\hline Cardiovascular source & 1 \\
\hline Skin source & 1 \\
\hline Mixed/unknown source & 2 \\
\hline Drug overdose & $5(6.8)$ \\
\hline Trauma & $1(1.4)$ \\
\hline Other/Mixed & $7(9.5)$ \\
\hline Unknown & $16(21.6)$ \\
\hline APACHE II score, mean [stdev] $(n=73)$ & $20.3[9.0]$ \\
\hline APACHE II score $\geq 25$ & $18(24.3)$ \\
\hline 30-day mortality $(n=69)$ & $19(27.5)$ \\
\hline Duration of hospital stay, median [range] days $(n=64)$ & $28.5(8-152)$ \\
\hline Duration of ITU stay, median [range] days $(n=64)$ & $14.5(2-68)$ \\
\hline Duration of mechanical ventilation, median [range] days $(n=57)$ & $12(1-61)$ \\
\hline \multicolumn{2}{|l|}{ Indication for BAL $(n=58)$} \\
\hline Suspected VAP & $29(39.2)$ \\
\hline Suspected aspiration pneumonia & $6(8.1)$ \\
\hline Suspected CAP & $6(8.1)$ \\
\hline Suspected HAP & $3(4.1)$ \\
\hline Combination of the above & $8(10.8)$ \\
\hline Other reason & $6(8.1)$ \\
\hline
\end{tabular}

median of 3 days [range 2-16 days]. However, once-daily microbiology consultation ward rounds occurred 7 days per week in the unit, with discussion of interim results before final reporting. Antimicrobial prescription and any changes in light of BAL fluid results were documented in the patient's medical records.

Antimicrobials were changed on the basis of the BAL fluid routine microbiological testing results in $35(60.3 \%)$ cases (Table S3); 19 (54.3\%) changes were de-escalations in number and/or spectrum of agent(s) and 14 (40.0\%) changes were escalations. In particular, 28 of the 35 antimicrobial changes are related to antibiotic changes. These decisions were reexamined in light of retrospective molecular test panel results to estimate the predicted benefits and risks of acting on molecular test identification results before culture results were available. In $15(53.6 \%)$ and $17(60.7 \%)$ cases respectively, acting on Unyvero P55 or in-house PCR panel identification results may have brought potential benefits as true-positive or true-negative results would have been available sooner (Table 5). However, there was a risk of a false-negative result being acted upon in six $(21.4 \%)$ cases with either molecular test. Risks and benefits were unclear in seven (25.0\%) and five (17.9\%) cases with Unyvero P55 and in-house PCR panels respectively. This was due to additional detections of unclear significance retrospectively or true-positive and false-negative detections for the same specimen.

\section{Discussion}

Microbiological investigation of lower respiratory tract infection in patients in the critical care setting would benefit from new methodologies with increased sensitivity and turnaround time. The aetiology of pneumonia is broad and few commercial respiratory panel assays are available with a sufficiently wide range of targets, particularly for the Gram-positive and 
Table 2 Detection of microorganisms in BAL fluids by routine microbiological culture and viral PCR $(n=74)$

\begin{tabular}{|c|c|}
\hline Microorganism & No. $(\%)$ \\
\hline \multicolumn{2}{|l|}{ Bacteria } \\
\hline Any bacteria & 48 (64.9) \\
\hline Gram-positive & $17(23.0)$ \\
\hline Staphylococcus aureus & 11 \\
\hline Staphylococcus epidermidis & 1 \\
\hline Staphylococcus haemolyticus & 1 \\
\hline Streptococcus agalactiae & 1 \\
\hline Streptococcus pneumoniae & 2 \\
\hline Enterococcus faecium & 1 \\
\hline Gram-negative & $42(56.8)$ \\
\hline Escherichia coli & 10 \\
\hline Haemophilus influenzae & 10 \\
\hline Klebsiella pneumoniae & 5 \\
\hline Pseudomonas aeruginosa & 5 \\
\hline Enterobacter cloacae complex & 4 \\
\hline Serratia marcescens & 2 \\
\hline Enterobacter aerogenes & 2 \\
\hline Klebsiella oxytoca & 1 \\
\hline Legionella maceachernii & 1 \\
\hline Neisseria meningitidis & 1 \\
\hline Citrobacter freundii & 1 \\
\hline Mycobacteria & $0(0.0)$ \\
\hline \multicolumn{2}{|l|}{ Fungi } \\
\hline Any fungi & $33(44.6)$ \\
\hline Candida albicans & 12 \\
\hline Candida glabrata & 1 \\
\hline Candida krusei & 1 \\
\hline Candida parapsilosis & 2 \\
\hline Candida tropicalis & 2 \\
\hline Yeasts - identification not required & 12 \\
\hline Pneumocystis jirovecii & 1 \\
\hline Aspergillus fumigatus & 4 \\
\hline \multicolumn{2}{|l|}{ Viruses } \\
\hline Any virus & $21(28.4)$ \\
\hline HSV-1 & 14 \\
\hline CMV & 6 \\
\hline Rhinovirus & 3 \\
\hline Influenza A & 2 \\
\hline Influenza B & 2 \\
\hline Any organism & 70 (94.6) \\
\hline$>1$ organism & $46(62.2)$ \\
\hline
\end{tabular}

Gram-negative bacteria seen frequently in ventilator and hospital-associated pneumonia $[3,4,15-17]$. In addition to this, rapid antibiotic resistance detection should enable more appropriate antibiotic selection to improve patient management, earlier antibiotic de-escalation to aid stewardship and earlier infection control interventions [18]. With this in mind, we tested the Unyvero P55 Pneumonia Cartridge assay retrospectively on a microbiologically well-characterised BAL specimen set to try to establish the potential benefits of using this approach in our ICU setting versus our in-house bacterial real-time multiplex PCR assays [5, 6].

As expected, the majority of our patients were undergoing BAL for suspected VAP, HAP or CAP and we isolated a wide range of Gram-positive and Gram-negative bacterial species from two-thirds of cases. The target coverage of cultured bacteria by the two molecular panels was higher at $89.9 \%$ for Unyvero P55 compared to $72.9 \%$ for the in-house PCR panel, and the few isolates cultured that were not in the Unyvero P55 panel were either rarely isolated pathogens or of doubtful significance. Fungal and viral pathogens absent from the Unyvero P55 panel were frequently identified by culture and routine PCR; however, the clinical significance of many of these was also unclear in this retrospective study. As expected, herpesvirus reactivation in this critical care cohort was common with HSV-1 and CMV, the most frequently detected viruses in BAL specimens. However, 3/14 patients with HSV-1 positive BAL specimens and 2/6 patients with CMV positive BAL specimens did receive specific antiviral treatment, indicating that significance was interpreted in the individual clinical context including concurrent viraemia.

We found that overall bacterial and target-specific sensitivity of the Unyvero P55 on BAL specimens was low at 56.9 and $63.5 \%$ respectively. In-house PCR assay performed better with overall and target-specific sensitivity of 63.2 and $83.7 \%$ respectively. For the Unyvero P55 test, we found that missed detections were predominantly bacterial targets which were present in the panel, whereas, for the in-house PCR assay, missed detections were mainly bacterial targets not present in the panel. Specificity of both tests was similar at $58.5 \%$ for the Unyvero P55 panel and 54.8\% for the in-house PCR panel, with additional organisms detected by both tests in $16.2 \%$ specimens. Sensitivity of Unyvero P55 has been assessed in two previous publications; our sensitivity estimate is more in line with that of Drick et al. [7] (66\%) who also used BAL specimens than that of Ozongwu et al. [19] (89\%) who predominantly used sputa and endotracheal specimens; therefore, specimen type may potentially affect the performance of the assay. Where additional detections were made by Unyvero P55 for targets present in our in-house molecular assay, we could corroborate $80 \%$ of them, suggesting that these were real additional positives, as also found in a previous study using molecular comparators [19]. An explanation is that the majority of patients in our study received antibiotics on the day of the BAL procedure and this was associated with reduced culture detection frequency, whereas molecular detection frequency was unaffected. Therefore, a bacterial culture may not be the appropriate "gold standard" for comparison. Many additional in-house PCR detections were negative by 
Table 3 Comparison of molecular panel tests to bacterial culture gold standard for bacterial identification in BAL specimens

\begin{tabular}{lllllllr}
\hline Test & $\begin{array}{l}\text { Culture }+ \\
\text { Test }+\end{array}$ & $\begin{array}{l}\text { Culture }+ \\
\text { Test }-\end{array}$ & $\begin{array}{l}\text { Culture }- \\
\text { Test }+\end{array}$ & $\begin{array}{l}\text { Culture }- \\
\text { Test }-\end{array}$ & Sensitivity (\%) & Specificity (\%) \\
\hline $\begin{array}{l}\text { Unyvero P55 } \\
\text { Overall }\end{array}$ & 33 & 25 & 17 & 24 & 56.9 & 58.5 & 66.0 \\
Unyvero P55 in-panel targets only & 33 & 19 & 17 & 24 & 63.5 & 59.0 \\
$\begin{array}{l}\text { In-house PCR panel } \\
\text { Overall }\end{array}$ & 36 & 21 & 19 & 23 & 63.2 & 58.5 & 54.8 \\
In-house PCR in-panel targets only & 36 & 7 & 19 & 23 & 83.7 & 54.8 & 65.5 \\
\hline
\end{tabular}

$P P V$ positive predictive value, $N P V$ negative predictive value

Unyvero P55, and this may reflect the higher sensitivity of inhouse PCR compared to Unyvero P55, as seen in culturepositive specimens.

The sensitivity of the Unyvero P55 panel for antibiotic resistance gene detection was low at $18.8 \%$, with particular problems detecting aminopenicillin, third-generation cephalosporin and macrolide/lincosamide resistance. In agreement with our study, Ozongwu et al. also found variable ability of the Unyvero P55 assay to detect antimicrobial resistance, despite excluding the results of tem, shv, ermB and sull gene detection. The existence of multiple genetic variants and the fact that mechanisms of resistance are also present in nonpathogenic respiratory tract microflora mean that accurate and relevant resistance gene detection direct from clinical specimens is not straightforward.

Diagnosis of VAP is in part reliant on a microbiological quantitative culture threshold of $>10$ colony forming units $(\mathrm{CFU}) / \mathrm{ml}[15]$ and we and others have demonstrated that molecular quantification of bacteria in BALs from VAP patients can be useful diagnostically $[16,20]$. However, the Unyvero P55 system is only indicative of bacterial load in a semiquantitative manner $(+,++$ or +++ read-outs per target) and therefore this aspect was not further analysed here. Ozongwu et al. [19] noted an $11 \%$ partial/complete failure rate with the Unyvero P55 assay, a phenomenon also reported in earlier studies using the previous version of the assay [21-24]. In our study, however, only 1/74 (1.4\%) of the Unyvero P55 tests was invalid, perhaps due to the difference between extraction of nucleic acids from BAL fluids compared to other specimen types such as sputa.

It was clear that routine BAL microbiological test results discussed on daily Microbiology ward rounds in the ICU contributed to patient management, with resulting changes to antimicrobials documented in $60 \%$ of cases. There were considerable potential benefits of using either molecular panel, in that true-negative and true-positive results would have been available much sooner in at least half of these cases, assuming a molecular assay turnaround time of 5 to $6 \mathrm{~h}$ compared to at least $24 \mathrm{~h}$ for preliminary culture results. However, negative results could not be relied upon due to the low sensitivity of the Unyvero P55 test and the small target range of the in-house bacterial PCR. In reality, therefore, if a molecular test was negative, results of culture would be awaited before any changes made. If a molecular test was positive, knowledge

Table 4 Comparison of Unyvero P55 panel antibiotic resistance gene detection to the phenotypic antibiotic susceptibility testing gold standard in BAL specimens

\begin{tabular}{|c|c|c|c|c|c|c|c|c|}
\hline Antibiotic/gene (s) & Resistant/P55 Pos & Resistant/P55 Neg & Suscept/P55 Pos & Suscept/P55 Neg & Sensitivity (\%) & Specificity (\%) & PPV & NPV \\
\hline $\begin{array}{l}\text { Aminopenicillin } \\
\text { tem/shv }\end{array}$ & 8 & 17 & 1 & 9 & 32.0 & 90.0 & 88.9 & 34.6 \\
\hline $\begin{array}{l}\text { 3rd-gen cephalosporin } \\
c t x-M\end{array}$ & 0 & 8 & 0 & 22 & 0.0 & 100.0 & N/A & 73.3 \\
\hline $\begin{array}{l}\text { Carbapenem } \\
\text { ndm/kpc/vim/imp/oxa }\end{array}$ & 0 & 3 & 0 & 26 & 0.0 & 100.0 & N/A & 89.7 \\
\hline $\begin{array}{l}\text { Fluoroquinolone } \\
\text { gyrA83/gyrA } 87\end{array}$ & 1 & 2 & 4 & 39 & 33.3 & 90.7 & 20.0 & 95.1 \\
\hline $\begin{array}{l}\text { Oxacillin } \\
m e c A / m e c C\end{array}$ & 0 & 1 & 1 & 9 & 0.0 & 90.0 & 0.0 & 90.0 \\
\hline $\begin{array}{l}\text { Macrolide/lincosamide } \\
\text { ermB }\end{array}$ & 0 & 8 & 0 & 7 & 0.0 & 100.0 & N/A & 46.7 \\
\hline Total & 9 & 39 & 6 & 112 & 18.8 & 94.9 & N/A & 74.2 \\
\hline
\end{tabular}

Suscept susceptible, $P P V$ positive predictive value, $N P V$ negative predictive value

$N / A$ not applicable — not possible to calculate value 
Table 5 Estimated potential benefits and risks of acting on results from molecular panel assays before culture results available $(n=28)$

\begin{tabular}{lll}
\hline & Unyvero P55 panel & In-house PCR panel \\
\hline Potential benefit (\%) & $15(53.6)$ & $17(60.7)$ \\
True-positive identification result obtained sooner & 9 & 12 \\
True negative result obtained sooner & 6 & 5 \\
Potential risk (\%) & $6(21.4)$ & $6(21.4)$ \\
False-negative result acted upon & 6 & 6 \\
Potential benefit/risk unclear (\%) & $7(25.0)$ & $5(17.9)$ \\
Additional positive identification result and/or combination of true-positive and false-negative results & 7 & 5 \\
\hline
\end{tabular}

of the identity of the organism may have been sufficient to enable faster antibiotic decisions, as found in a previous BAL study in an ICU [25]. Given the low sensitivity and variable negative predictive value of Unyvero P55 antibiotic resistance gene detection, this aspect of the assay conferred no additional benefit and potentially some risk.

The strengths of this study were its use of a large and microbiologically well-characterised BAL specimen set, comparative molecular testing and corresponding detailed antimicrobial prescribing information. A previous study showed that Unyvero P55 enabled rapid antibiotic modification in nosocomial pneumonia, but was limited by low frequency of culture positivity, low numbers of specimens and BAL specimens in particular and lack of a molecular comparator test [26]. In that study, sensitivity and specificity were not specifically calculated, but Unyvero P55 identified significantly more bacterial co-infections than routine culture. A recent study of Unyvero P55 on BAL specimens in lung transplant recipients again had low sample numbers but reported a sensitivity of $66 \%$ with specificity of $100 \%$ as there were no additional PCR positive culture negative cases [7]. Given that this study was conducted in an outpatient setting, it was unclear how the results of molecular testing would have contributed to patient management and antibiotic resistance gene detection was not assessed [7]. A large UK study reported Unyvero P55 sensitivity of $89 \%$ and specificity of $95 \%$ on mainly sputa and endotracheal aspirates from a mixed population of CAP, HAP and VAP patients, but did not assess potential clinical impact [19].

The present study is limited however by its retrospective nature. Degradation and dilution of nucleic acids by storage may have reduced the pathogen load in the specimen set, giving a more severe test of sensitivity than in a prospective study with fresh specimens. However, on a target-specific basis, the sensitivity of the Unyvero P55 panel appeared to be lower than that of the in-house panel, indicating that the wider target range and ease of use may come at the expense of some sensitivity relative to in-house multiplex real-time PCR. Antibiotic prescription data and decision-making were also analysed retrospectively, although this information was well documented in clinical notes made at the time. Inferring potential benefits and risks is difficult retrospectively but we believe it gives a pragmatic insight into how useful these tests would be in our setting. It is possible that in a setting with a lower culture-positive rate, higher antibiotic resistance or lacking regular Clinical Microbiologist input, there may be greater benefits from the Unyvero P55 panel [26].

For now, there is still a clear need to perform traditional microbiological culture of BAL fluids from patients in the ICU, at the very least, to confirm negative molecular tests and to detect antibiotic resistance reliably. We are, however, still missing potentially $16 \%$ of cases of bacterial infection with culture alone, and possibly more where molecular targets are still required for particular bacterial species, so there is further scope to improve patient management and antibiotic stewardship. Exactly which molecular assay will prove the most useful in this regard will depend very much on the individual setting; in our hands, a narrower but more sensitive inhouse PCR panel was a more pragmatic and cost-effective solution. It is clear that at present, however, molecular tests may only be a useful adjunct to routine testing.

Acknowledgements Unyvero reagents and instruments were provided by the manufacturer (Curetis AG).

\section{References}

1. Chen JHK, Lam HY, Yip CCY, Wong SCY, Chan JFW, Ma ESK, Cheng VCC, Tang BSF, Yuen KY (2016) Clinical evaluation of the new high-throughput Luminex NxTAG respiratory pathogen panel assay for multiplex respiratory pathogen detection. J Clin Microbiol 54(7):1820-1825

2. Andrews D, Chetty Y, Cooper BS, Virk M, Glass SK, Letters A, Kelly PA, Sudhanva M, Jeyaratnam D (2017) Multiplex PCR point of care testing versus routine, laboratory-based testing in the treatment of adults with respiratory tract infections: a quasi-randomised study assessing impact on length of stay and antimicrobial use. BMC Infect Dis 17(1):671

3. Baudel JL, Tankovic J, Dahoumane R, Carrat F, Galbois A, AitOufella H, Offenstadt G, Guidet B, Maury E (2014) Multiplex PCR performed of bronchoalveolar lavage fluid increases pathogen identification rate in critically ill patients with pneumonia: a pilot study. Ann Intensive Care 4:35 
4. Ullberg M, Lüthje P, Mölling P, Strålin K, Özenci V (2017) Broadrange detection of microorganisms directly from bronchoalveolar lavage specimens by PCR/electrospray ionization-mass spectrometry. PLoS One 12(1):e0170033

5. Gadsby NJ, McHugh MP, Russell CD, Mark H, Conway Morris A, Laurenson IF, Hill AT, Templeton KE (2015) Development of two real-time multiplex PCR assays for the detection and quantification of eight key bacterial pathogens in lower respiratory tract infection. Clin Microbiol Infect 21:788.e1-788.13

6. Gadsby NJ, Russell CD, McHugh MP, Mark H, Conway Morris A, Laurenson IF, Hill AT, Templeton KE (2016) Comprehensive molecular testing for respiratory pathogens in community-acquired pneumonia. Clin Infect Dis 62(7):817-823

7. Drick N, Seeliger B, Greer M, Bollmann BA, Ziesing S, Welte T, Gottlieb J (2018) DNA-based testing in lung transplant recipients with suspected non-viral lower respiratory tract infection: a prospective observational study. Transpl Infect Dis 20(1):e12811

8. Public Health England (2018) Investigation of bronchoalveolar lavage, sputum and associated specimens. UK standards for Microbiology Investigations. B 57 Issue 3. https://www.gov.uk/ government/publications/smi-b-57-investigation-ofbronchoalveolar-lavage-sputum-and-associated-specimens. Accessed 20 Dec 2018

9. Heim A, Ebnet C, Harste G, Pring-Akerblom P (2003) Rapid and quantitative detection of human adenovirus by real-time PCR. J Med Virol 70:228e39

10. Templeton KE, Scheltinga SA, Sillekens P, Crielaard JW, van Dam AP, Goossens H, Claas EC (2003a) Development and clinical evaluation of an internally controlled, single-tube multiplex real-time PCR assay for detection of legionella pneumophila and other legionella species. J Clin Microbiol 41(9):4016e21

11. Templeton KE, Scheltinga SA, Graffelman AW, Van Schie JM, Crielaard JW, Sillekens P, Van Den Broek PJ, Goossens H, Beersma MF, Claas EC (2003b) Comparison and evaluation of real-time PCR, real-time nucleic acid sequence-based amplification, conventional PCR, and serology for diagnosis of mycoplasma pneumoniae. J Clin Microbiol 41:4366e71

12. Scheltinga SA, Templeton KE, Beersma MF, Claas EC (2005) Diagnosis of human metapneumovirus and rhinovirus in patients with respiratory tract infections by an internally controlled multiplex real-time RNA PCR. J Clin Virol 33:306e11

13. Templeton KE, Scheltinga SA, Beersma MF, Kroes AC, Claas EC (2004) Rapid and sensitive method using multiplex real-time PCR for diagnosis of infections by influenza A and influenza B viruses, respiratory syncytial virus, and parainfluenza viruses $1,2,3$, and 4 . J Clin Microbiol 42:1564e9

14. Linssen CF, Jacobs JA, Beckers P, Templeton KE, Bakkers J, Kuijper EJ, Melchers WJ, Drent M, Vink C (2006) Interlaboratory comparison of three different real-time PCR assays for the detection of Pneumocystis jiroveci in bronchoalveolar lavage fluid samples. J Med Microbiol 55:1229-1235

15. Chastre J, Fagon JY (2002) Ventilator-associated pneumonia. Am J Respir Crit Care Med 165(7):867-903

16. Clavel M, Barraud O, Moucadel V, Meynier F, Karam E, Ploy MC, François B, VALIBI study group (2016) Molecular quantification of bacteria from respiratory samples in patients with suspected ventilator-associated pneumonia. Clin Microbiol Infect 22(9): 812.e1-812.e7

17. Pulido MR, Moreno-Martínez P, González-Galán V, Fernández Cuenca F, Pascual Á, Garnacho-Montero J, Antonelli M, Dimopoulos G, Lepe JA, McConnell MJ, Cisneros JM, MagicBullet Working Group (2018) Application of BioFire FilmArray blood culture identification panel for rapid identification of the causative agents of ventilator-associated pneumonia. Clin Microbiol Infect 24(11):1213.e1-1213.e4

18. Perez KK, Olsen RJ, Musick WL, Cernoch PL, Davis JR, Land GA, Peterson LE, Musser JM (2013) Integrating rapid pathogen identification and antimicrobial stewardship significantly decreases hospital costs. Arch Pathol Lab Med 137(9):1247-1254

19. Ozongwu C, Personne Y, Platt G, Jeanes C, Aydin S, Kozato N, Gant V, O'Grady J, Enne VI (2017) The Unyvero P55 'sample-in, answer-out' pneumonia assay: a performance evaluation. Biomol Detect Quantif 13:1-6

20. Conway Morris A, Gadsby N, McKenna JP, Hellyer TP, Dark P, Singh S, Walsh TS, McAuley DF, Templeton K, Simpson AJ, McMullan R (2017) 16S pan-bacterial PCR can accurately identify patients with ventilator-associated pneumonia. Thorax 72:10461048

21. Schulte B, Eickmeyer H, Heininger A, Juretzek S, Karrasch M, Denis O, Roisin S, Pletz MW, Klein M, Barth S, Lüdke GH, Thews A, Torres A, Cillóniz C, Straube E, Autenrieth IB, Keller PM (2014) Detection of pneumonia associated pathogens using a prototype multiplexed pneumonia test in hospitalized patients with severe pneumonia. PLoS One 9(11):e110566

22. Kunze N, Moerer O, Steinmetz N, Schulze MH, Quintel M, Perl T (2015) Point-of-care multiplex PCR promises short turnaround times for microbial testing in hospital-acquired pneumonia-an observational pilot study in critical ill patients. Ann Clin Microbiol Antimicrob 14:33

23. Personne Y, Ozongwu C, Platt G, Basurto-Lozada P, Shamin M, Gant VA, Zumla A, Enne VI (2016) 'Sample-in, answer-out'? Evaluation and comprehensive analysis of the Unyvero P50 pneumonia assay. Diagn Microbiol Infect Dis 86(1):5-10

24. Papan C, Meyer-Buehn M, Laniado G, Nicolai T, Griese M, Huebner J (2018) Assessment of the multiplex PCR-based assay Unyvero pneumonia application for detection of bacterial pathogens and antibiotic resistance genes in children and neonates. Infection 46(2):189-196

25. Sircar M, Ranjan P, Gupta R, Jha OK, Gupta A, Kaur R, Chavhan N, Singh M, Singh SK (2016) Impact of bronchoalveolar lavage multiplex polymerase chain reaction on microbiological yield and therapeutic decisions in severe pneumonia in intensive care unit. J Crit Care 31(1):227-232

26. Jamal W, Al Roomi E, AbdulAziz LR, Rotimi VO (2014) Evaluation of Curetis Unyvero, a multiplex PCR-based testing system, for rapid detection of bacteria and antibiotic resistance and impact of the assay on management of severe nosocomial pneumonia. J Clin Microbiol 52(7):2487-2492

Publisher's note Springer Nature remains neutral with regard to jurisdictional claims in published maps and institutional affiliations. 